\title{
Studies on the Thermo-Mechanical Properties of Gelatin Based Films Using 2-Hydroxyethyl Methacrylate by Gamma Radiation
}

\author{
Haydar U. Zaman*, Mubarak A. Khan, Ruhul A. Khan \\ Radiation and Polymer Chemistry Laboratory, Institute of Nuclear Science and Technology, Bangladesh Atomic Energy, Dhaka, \\ Bangladesh. \\ Email: "haydar_zaman@yahoo.com
}

Received October $15^{\text {th }}$, 2011; revised November 29 ${ }^{\text {th }}, 2011$; accepted December $18^{\text {th }}, 2011$.

\begin{abstract}
Gelatin films were prepared by casting. Tensile strength (TS) and elongation at break (Eb) of the gelatin films were found to be $46 \mathrm{MPa}$ and 3.5\%, respectively. Effect of gamma radiation (Co-60) on the thermo-mechanical properties of the gelatin films was studied. 2-hydroxyethyl methacrylate (HEMA) was added to the gelatin during casting varying (10\% - 30\% by weight) and found to increase the TS significantly. Then the films were irradiated and found further increase of TS. Thermo-mechanical properties of HEMA blended gelatin films were compared with those of the pure gelatin films. The coefficient of thermal expansion of the gelatin/HEMA films were also measured using thermo mechanical analyzer and found opposite trend with comparison of glass point.
\end{abstract}

Keywords: Gelatin; HEMA; Gamma Irradiation; Thin Film

\section{Introduction}

Gelatin is a relatively low cost protein, industrially produced all over the world and that have excellent film forming properties. Mainly because of that, this protein is being extensively explored in edible and/or biodegradable films production and characterization studies, pure [1-6], or blended with other biopolymers [7,8]. Gelatin is a polymer, as produced by the partial hydrolysis of collagen derived from the skin, white connective tissues, and bones of animals. Being derivative of protein, it is used in food, cosmetics, pharmaceuticals and photographic industries for its gel forming abilities, non toxicity and cheap production cost. In pharmaceuticals, gelatin is used as capsule shell manufacturing raw material for controlled drug release. Because of various potential uses of gelatin, it has been considered worthwhile to modify gelatin to enable improved or alternative applications [9].

Gelatin is a unique polymer comprising multifunctionalities like gelling, thickening, water-binding, emulsifying stabilizing, foaming, film forming and fining characteristics. It forms thermo reversible gels through the formation of hydrogen bond stabilized triple helices when its solution is cooled. Again on heating it melts above $40^{\circ} \mathrm{C}$. Hydrogen bond stabilization is followed by rearrangement of individual molecular chains into ordered, helical arran-

${ }^{*}$ Corresponding author. gement, or collagen fold and association of two or three ordered segments to create crystallites [10]. Besides, gelatin, similar to synthetic high polymers, shows a rather wide molecular weight distribution [11]. It is soluble in water and in aqueous solutions of polyhydric alcohols such as glycerol and propylene glycol and also hydrogen-bonding organic solvents like acetic acid, trifluoroethanol, and formamide. Gelatin is practically insoluble in less polar organic solvents such as acetone, carbon tetrachloride, dimethylformamide and most other non polar organic solvents. There are limitless possibilities for modifying the properties of gelatin because the number of bi and polyfunctional organic and inorganic compounds that can interact with the particular gelatin functions is very large indeed [12]. Chemical modifications, aiming at an increase in the degree of protein cross-linking depend on the reactivity of the protein constituents, the specificity of the modifying agent [13], the amino acid composition, the reactivity of amino acid and the tri-dimensional structure of the protein molecule. Generally, chemical reactivity of proteins depends on the side chain, the amino acid composition and the free amino and carboxyl groups [14]. The most reactive protein groups are serine (primary-OH), hydroxiproline (secondary-OH), threonine (secondary-OH), tyrosine (phenolic-OH), aspartic acid (-COOH), glutamic acid (-COOH), lysine (-NH2) and arginine (-C(:NH).NH2) [15]. Crosslinking of gelatin macromolecules is known to increase the 
viscosity of the gelatin solutions, strength and melting points of gelatin gels. The cross-linking of gelatin matrix by chemical means is used extensively in photographic products, and this so-called hardening permanently reduces the solubility of gelatin. It is important that graft gelatin copolymers retain the valuable properties of the parent gelatin, the ability to form gels and helices and the high heat resistance. The synthesis, structure, thermo-physical and physico-mechanical properties of graft gelatin copolymers have been studied in detail [16-22]. On heating, gelatin undergoes not only structural and mechanical but also physico-chemical transformations such as partial or complete loss of solubility in water. Stejskal et al. [23] observed that when methyl methacrylate is polymerized in aqueous medium in the presence of gelatin, gelatin graft copolymer macromolecules are formed. Little work on radiation-induced simultaneous copolymerization with acrylic monomers associated as blend with gelatin is reported. In the present study we reported on simultaneous copolymerization of 2-hydroxyethyl methacrylate with gelatin using blending and casting method, where simultaneous evaporation at room temperature was driving force. Later the films were subjected to gamma irradiation. The mechanical and thermo-mechanical properties of the films were analyzed.

\section{Experimental}

\subsection{Materials}

Pharmaceutical grade gelatin was collected from Global Capsules of Opsonin Pharma Ltd. HEMA was supplied by E. Merk, Germany.

\subsection{Methods}

\subsubsection{Preparation of Gelatin Films}

Granules of gelatin (15 g) were dissolved in hot water and different percentages of HEMA ( $10 \%$ - 30\% by wt) were mixed for different formulations and heated at $60^{\circ} \mathrm{C}$ for about half an hour until it reaches a viscous state. Three formulations were prepared named as B1 - B3; their compositions are given in Table 1. The solution was then cast on to the plastic covered uniform surface to form film under room conditions. It was then dried at room temperature.

Table 1. Composition of HEMA blended gelatin formulations $(\% \mathrm{w} / \mathrm{w})$ for film preparation.

\begin{tabular}{cccc}
\hline \multirow{2}{*}{ Formulations } & \multicolumn{3}{c}{ Compositions (\%) } \\
\cline { 2 - 4 } & HEMA & Water & Gelatin \\
\hline B1 & 10 & 75 & 15 \\
B2 & 20 & 65 & 15 \\
B3 & 30 & 55 & 15 \\
\hline
\end{tabular}

The dried films (about $0.30 \mathrm{~mm}$ thickness) were peeled off and cut into small pieces of length $70 \mathrm{~mm}$ and width 10 $\mathrm{mm}$ using conventional scissors. HEMA-blended gelatin films were subjected to irradiation with gamma radiation using a $\mathrm{Co}^{60}$ source (25 $\mathrm{kCi}$ model gamma beam 650 is loaded with source GBS-98 that comprises of 36 double encapsulated capsules. Type C-252 loaded with $\mathrm{Co}^{60}$ pellets). The gelatin films were subjected to irradiation with different gamma doses (50 - $500 \mathrm{krad})$ at a dose rate of 350 $\mathrm{krad} / \mathrm{hr}$ using $\mathrm{Co}^{60}$ gamma source. The relative humidity was around $78 \%$ and the temperature was $32^{\circ} \mathrm{C}$. These samples were stored in a laminated poly ethylene bag until testing.

\subsubsection{Mechanical Test}

Tensile properties such as tensile strength (TS) and percent elongation at break (Eb) of the cured films were measured with Universal Testing Machine (Hounsfield Series $\mathrm{S}$, UK) with a cross head speed of $10 \mathrm{~mm} / \mathrm{min}$. The load range of $500 \mathrm{~N}$ and the gauze length $20 \mathrm{~mm}$ were used throughout the experiment. Four different blends with different concentrations of HEMA in gelatin were analyzed using universal testing machine. But higher compositions of HEMA films are softer and can easily absorb moisture. And due to radiation they became brittle. So we investigated the physico-mechanical properties of $10 \%, 20 \%$, $30 \%$ HEMA containing gelatin film at $65 \%$ relative humidity at room temperature to enable identical moisture content.

\subsubsection{Thermo-Mechanical Analysis}

On-set of melting, glass point, off-set of melting and linear coefficient of thermal expansion were measured for all the films using Thermo-mechanical Analyzer (Liensis 200) with an efficiency of \pm 3 degree centigrade.

\section{Results and Discussion}

\subsection{Mechanical Properties of Irradiated and Non Irradiated Films}

Tensile strength (TS) is very important in selecting diverse application of polymer. The results of TS values of the non irradiated and irradiated films are plotted in Figure 1 against total gamma radiation dose for gelatin (G), 10\% (B1), 20\% (B2) and 30\% (B3) HEMA blended gelatin film produced by blending. It is observed that with the loading of HEMA the TS significantly decreased which may be HEMA is acting as filler between inter molecular and inter chain spaces hindering the helix structure of film . But, due to irradiation the tensile strength was improved up to some radiation dose and above that dose it was decreased for almost all compositions. In case of pure gelatin film tensile strength value was also increased with the increase of radiation doses and attains a maximum at $100 \mathrm{krad}$ dose 


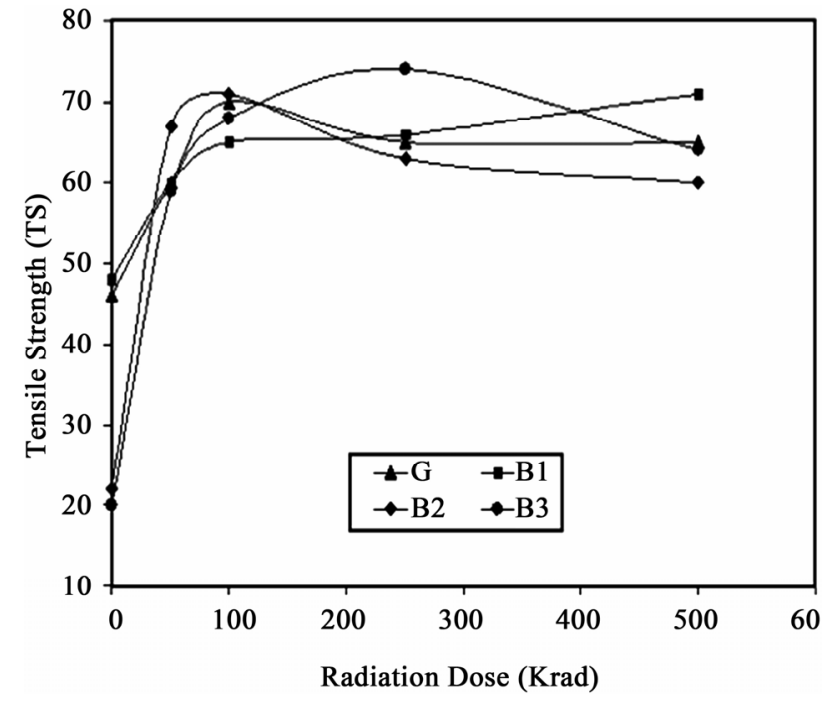

Figure 1. Tensile strength against radiation dose of gelatin (G) and HEMA blended gelatin films (B1, B2 and B3).

and then decreases with increasing radiation doses. Higher gamma radiation dose may have caused degradation of the polymer chains and the film became hard and brittle whilst at lower doses cross linking may have dominated over chain seasoning.

On the other hand, in case of gelatin/HEMA blend biofilm, tensile strength value reaches a maximum at a dose of $250 \mathrm{krad}$ and then decreased further with increasing gamma dose as well as gelatin concentration. When the gelatin/HEMA film subjected to the radiation, acrylic double bond from HEMA and gelatins functional groups might have initiated to form cross linked network. So, tensile strength value increases with radiation, but higher radiation doses might have caused chain scission due to the breaking of the polymer chains. The probable reaction is shown in Scheme 1. So, at higher radiation doses tensile strength decreased. From the Figure 1, it is clear that tensile strength value of gamma treated film is higher than that of untreated film. Highest tensile strength was found for 30\% HEMA containing gelatin film at 250 krad dose and was found to be $270 \%$ higher than the non-irradiated sample of same composition and with respect to pure gelatin film it was $23.3 \%$ higher.

\section{Elongation at Break (\% Eb)}

The results of elongation at break (\%) of the non irradiated and irradiated films against total gamma radiation dose are plotted in Figure 2 for Gealtin (G), 10\% (B1), 20\% (B2) and 30\% (B3) HEMA containing gelatin films produced by blending. From the Figure 2, it is observed that percent elongation at break increases for non-irradiated samples drastically due to incorporating HEMA. From the figure it is also observed that for non-irradiated film the highest elongation at break was found to be $32 \%$ for $20 \%$

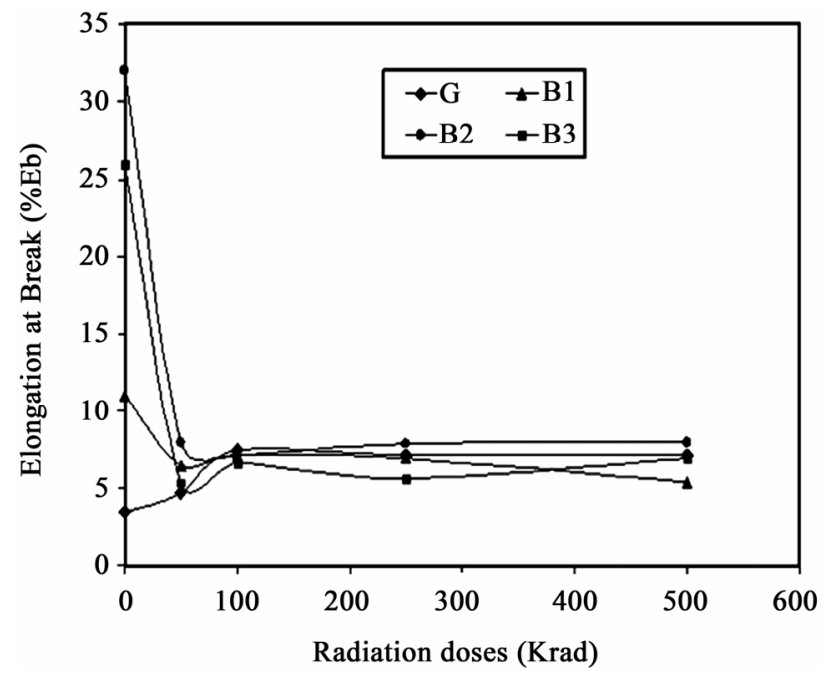

Figure 2. Elongation at Break against radiation dose of gelatin (G) and HEMA blended gelatin films (B1, B2 and B3).

HEMA containing gelatin film which is $28.5 \%$ higher than pure gelatin film.

This is may be caused by plasticizing effect of HEMA over gelatin which made the film comparatively softer. Elongation is an important mechanical property in the application of polymer. Observing the figure, it is found that the value of elongation is decreasing with the increasing value of radiation intensity though tensile strength was increased and so happened due to cross linking. But the elongation at break increased due to incorporation of HEMA comparing with that of pure gelatin. Gelatin film containing 30\% HEMA at 250krad radiation dose, at which highest tensile strength was found, was also found to have $5.6 \%$ elongation at break. This amount is higher than that of pure gelatin film.

\subsection{Thermo Mechanical Analysis (TMA)}

\subsubsection{Glass Point}

Graphs, showing amount of probe movement vs. temperature, for glass point analysis is shown in Figures 3-6. Glass point is plotted against composition of HEMA in gelatin films have been plotted in Figure 7. An almost linear trend in increasing glass point with increasing amount of HEMA blended with gelatin has been observed. The highest glass point observed was $86.4^{\circ} \mathrm{C}$ for gelatin/HEMA biofilm containing 30\% HEMA. The change in molecular interaction and different thermal response of the monomer and gelatin might have shifted the glass point of film.

\subsubsection{Coefficient of Thermal Expansion (CTE)}

CTE is an important quality as it indicates its topological and morphological change when subjected to change in temperature. CTE for different composition of gelatin/HEMA film is shown against temperature in Figure 8. Again 


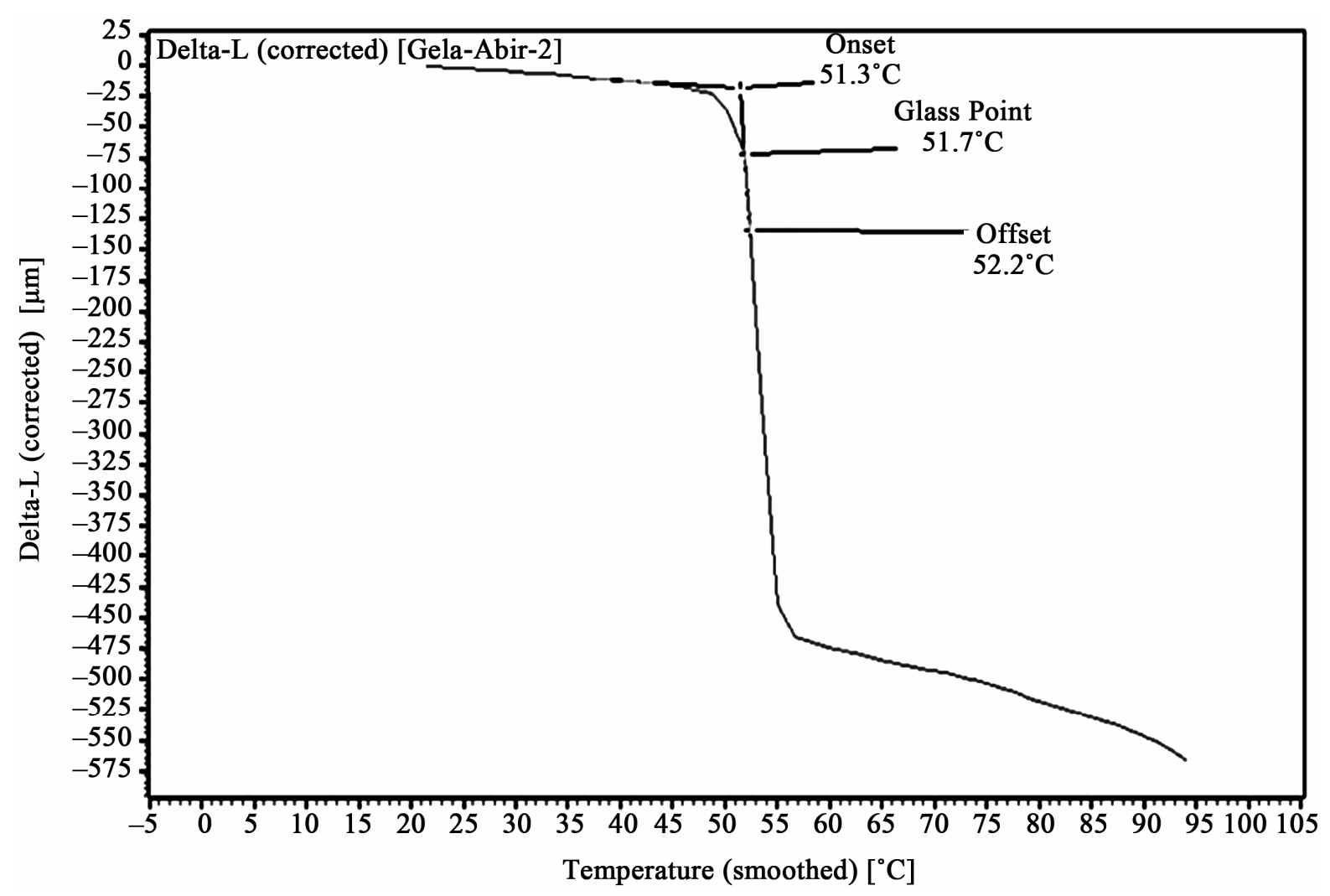

Figure 3. TMA of untreated pure gelatin film.

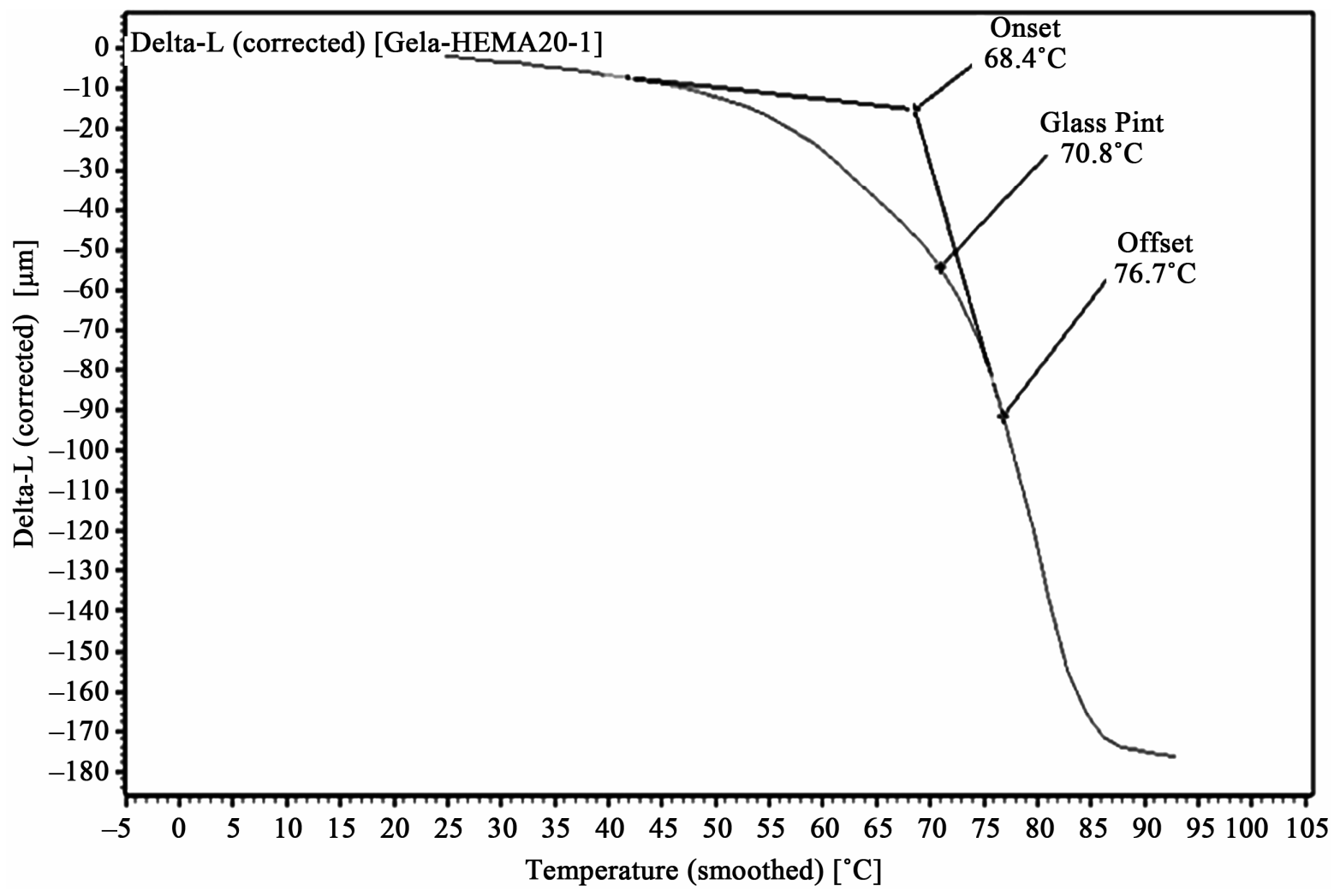

Figure 4. TMA of $10 \%$ HEMA/gelatin film. 


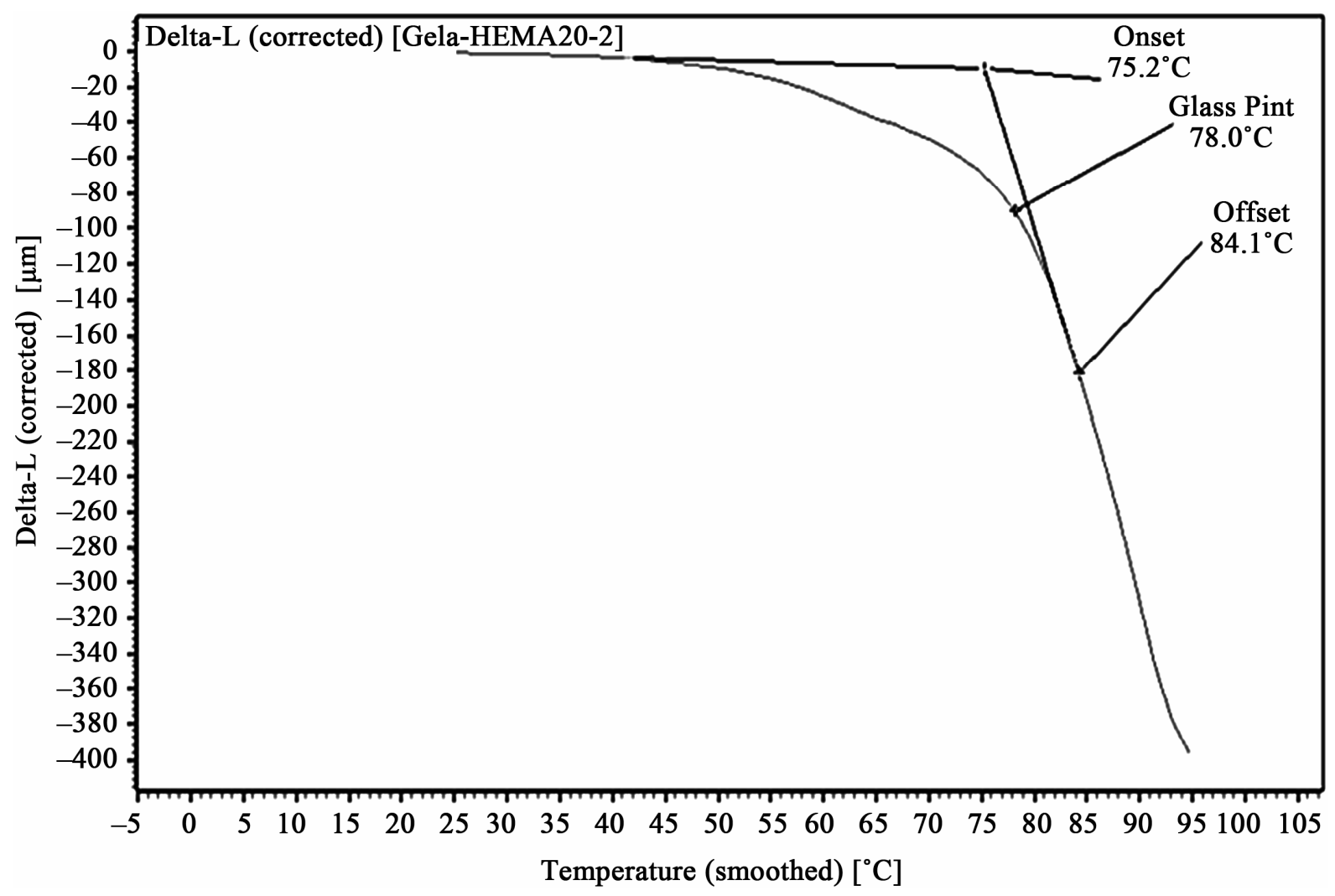

Figure 5. TMA of $20 \%$ HEMA/gelatin film.

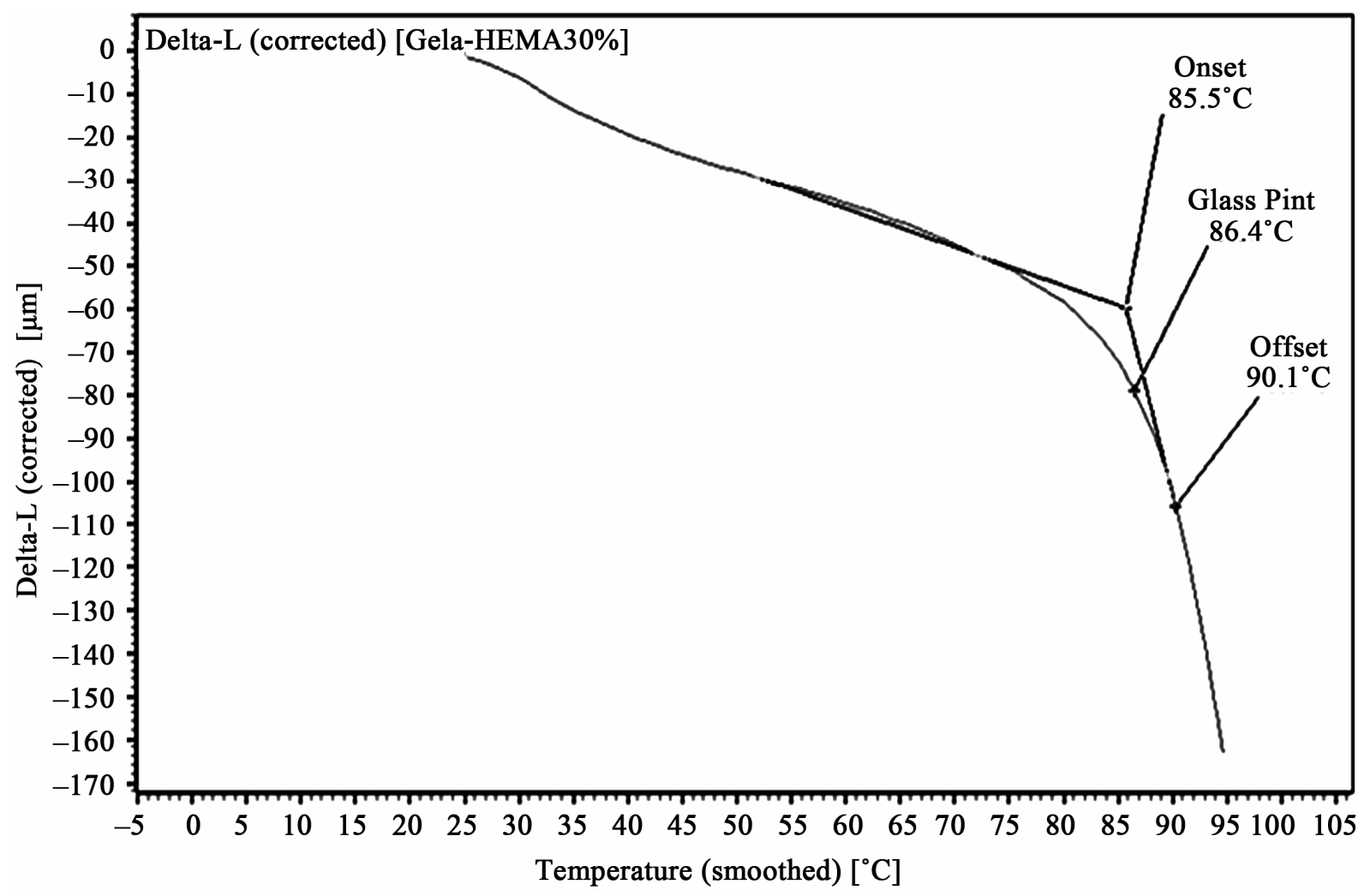

Figure 6. TMA of $30 \%$ HEMA/gelatin film. 


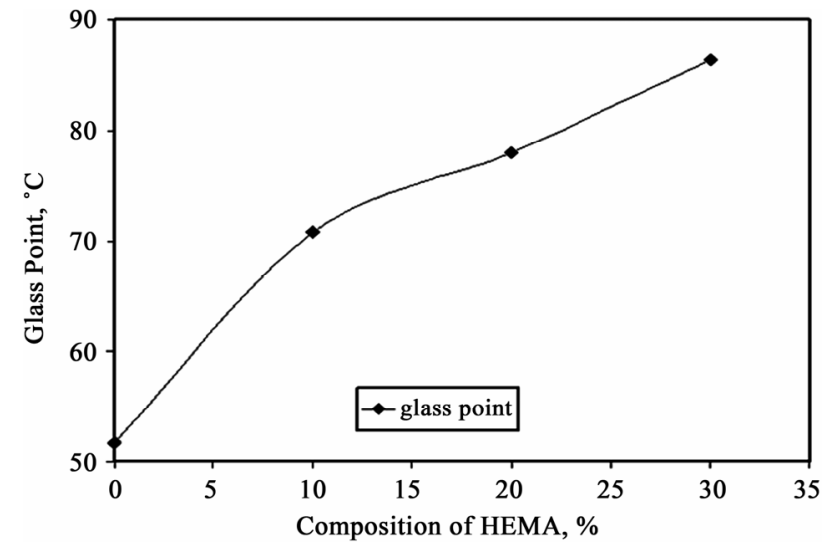

Figure 7. Glass point versus composition of HEMA in gelatin film.

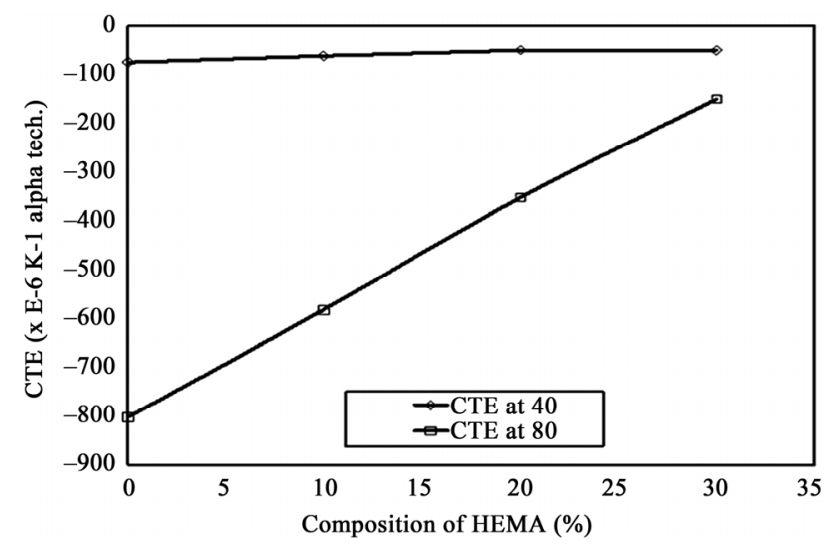

Figure 8. CTE for different composition of gelatin/HEMA film against temperature.

CTE of control specimen and gelatin/HEMA biofilms of different compositions at $40^{\circ} \mathrm{C}$ and $80^{\circ} \mathrm{C}$ have been plotted against respective percentages of HEMA present in films in Figure 8. Opposite trend to glass point was observed, that is, with increasing amount of HEMA the CTE was decreased almost linearly and the increase in CTE at $80^{\circ} \mathrm{C}$ was more rapid. Gelatin has a negative coefficient of thermal expansion while acrylic polymers have a positive CTE. So, the decreased contraction, in other words negative expansion was quite expectable. The difference between CTE at $40^{\circ} \mathrm{C}$ and $80^{\circ} \mathrm{C}$ was found lowest for gelatin/HEMA biofilm containing 30\% HEMA. Thus film containing 30\% HEMA would go through less physical distortion while exposed to higher temperature.

\section{Conclusions}

During the study physico mechanical and thermo mechanical properties of irradiated and non-irradiated pure gelatin and HEMA/gelatin film have been studied. Due to incorporating HEMA and gamma radiation the tensile strength was found to be improved and for 30\% HEMA /gelatin film irradiated at $250 \mathrm{krad}$, it was 23\% higher than pure gelatin film. Also the elongation at break was improved to $5.6 \%$. For 30\% HEMA containing unirradiated film it was $32 \%$. The thermo mechanical properties have been drastically improved due to HEMA content in films. The glass point increased almost linearly due to increasing HEMA content in gelatin film. Gelatin showed contraction on heating, hence negative thermal expansion was found. The coefficient of thermal expansion showed the opposite trend as it was found to be decreased with temperature but it was comparatively smaller for films containing higher percentages of HEMA. That indicates much thermal stability due to blending HEMA with gelatin.

\section{REFERENCES}

[1] L. T. Lim, Y. Mine and A. Tung, "Barrier and Tensile Properties of Transglutaminase Cross-linked Gelatin Films as Affected by Relative Humidity, Temperature, and Glycerol Content,” Journal of Food Science, Vol. 64, No. 4, 1999, pp. 616-622. doi:10.1111/j.1365-2621.1999.tb15096.x

[2] P. J. A. Sobral, F. C. Menegalli, M. D. Hubinger and M. A. Roques, "Mechanical, Water Vapor Barrier and Thermal Properties of Gelatin Based Edible Films," Food Hydrocolloids, Vol. 15, No. 4-6, 2001, pp. 423-432. doi:10.1016/S0268-005X(01)00061-3

[3] R. A. Carvalho and C. R. F. Grosso, "Characterization of Gelatin Based Films Modified with Transglutaminase, Glyoxal and Formaldehyde," Food Hydrocolloids, Vol. 18, No. 5, 2004, pp. 717-726. doi:10.1016/j.foodhyd.2003.10.005

[4] P. Bergo, R. A. Carvalho, A. C. S. Vadala, V. C. I. Guevara and P. J. A. Sobral, "Physical Properties of Gelatin Films Plasticized with Glycerol, Studied by Spectroscopic Methods," Materials Science Forum, Vol. 636-637, 2010, pp. 753-758. doi:10.4028/www.scientific.net/MSF.636-637.753

[5] F. M. Vanin, P. J. A. Sobral, F. C. Menegalli, R. A. Carvalho and A. M. Q. B. Habitante, "Effects of Plasticizers and Their Concentrations on Thermal and Functional Properties of Gelatin-Based Films,” Food Hydrocolloids, Vol. 19, No. 5, 2005, pp. 899-907. doi:10.1016/j.foodhyd.2004.12.003

[6] P. V. A. Bergo, R. A. Carvalho, P. J. A. Sobral, F. R. S. Bevilacqua, J. K. C. Pinto and J. P. Souza, "Microwave Transmittance in Gelatin-Based Films," Measurement Science and Technology, Vol. 17, No. 12, 2006, pp. 32613264. doi:10.1088/0957-0233/17/12/010

[7] I. Arvanitoyannis, A. Nakayama, S.-I. Aiba and N. Yamamoto, "Edible Films Made from hydroxypropyl Starch and Gelatin and Plasticized by Polyols and Water," Carbohydrate Polymers, No. 36, No. 2-3, 1998, pp. 105-119.

[8] Gelatin, Standard Methods for Sampling and Testing Gelatins, Gelatin Manufacturers' Institute of America, Inc., New York, 1986,

[9] A. Eis, "The Macromolecular Chemistry of Gelatin," 
Academic Press, New York, 1964.

[10] P. V. Kozlov and G. I. Burdygina, "The Structure and Properties of Solid Gelatin and the Principles of Their Modification,” Polymer, Vol. 24, No. 6, 1983, pp. 651666. doi:10.1016/0032-3861(83)90001-0

[11] S. S. Wong and L. J. C. Wong, "Chemical Crosslinking and the Stabilization of Proteins and Enzymes," Enzyme and Microbial Technology, Vol. 14, No. 11, 1992, pp. 866874. doi:10.1016/0141-0229(92)90049-T

[12] G. E. Means and R. E. Feeney, "Chemical Modification of Proteins,” Holden-Day Inc., Vol. 3, San Francisco, 1971, pp. 35-51.

[13] C. A. Finch, "Chemistry and Technology of Water Soluble Polymers,” in: C. A. Finch, Ed., Plenum Press, Vol. 5, New York, 1983, pp. 81-112.

[14] C. I. Burdygina, L. Ya. Chenborisova, A. I. Maklakov and P.V. Kozlov, "International Symposium on Macromolecular Chemistry,” Taskent Abstracts, Vol. 5, 1978, p. 170.

[15] S. Sarkar, A. Chourasia, S. Maji, S. Sadhukhan, S. Kumar and B. Adhikari, "Synthesis and Characterization of Gelatin Based Polyester Urethane Scaffold,” Ind. Acad. Sci., Vol. 29, 2006, pp. 475-484.

[16] H. Babin and E. Dickinson, "Influence of Transglutaminase Treatment on the Thermoreversible Gelation of Gelatin,” Food Hydrocolloids, Vol. 15, No. 3, 2001, pp. 271-276. doi:10.1016/S0268-005X(01)00025-X

[17] S. Sterman and J. G. Marsden, "The Effect of Silane Coupling Agents in Improving the Properties of filled or Reinforced Thermoplastics," Polymer Engineering \& Science, Vol. 6, No. 2, 2004, pp. 97-112.

\section{doi:10.1002/pen.760060203}

[18] C. Jo, H. Kang, N.Y. Lee, J. H. Kwon and M. W. Byun, "Pectin- and Gelatin-Based Film: Effect of Gamma Irradiation on the Mechanical Properties and Biodegradation," Radiation Physics and Chemistry, Vol. 72, No. 6, 2005, pp. 745-750. doi:10.1016/j.radphyschem.2004.05.045

[19] A. Banihashemi, H. Hazarkhani and A. Abdolmaleki, "Efficient and Rapid Synthesis of Polyureas and Polythioureas from the Reaction of Urea and Thiourea with Diamines under Microwave Irradiation,” Journal of Polymer Science Part A: Polymer Chemistry, Vol. 42, No. 9, 2004, pp. 2106-2111. doi:10.1002/pola.20060

[20] P. Haque, A. I. Mustafa and M. A. Khan, "Effect of CrossLinking Monomers on the Physico-Mechanical and Degradation Properties of Photografted Chitosan Film," Carbohydrate Polymers, Vol. 68, No. 1, 2007, pp. 109-115.

[21] C. Dai, Y. Chen and M. Liu, "Thermal properties MeasUrements of Renatured Gelatin Using Conventional and Temperature Modulated Differential Scanning Calorimetry," Journal of Applied Polymer Science, Vol. 99, No. 4, 2006, pp. 1795-1801. doi:10.1002/app.22711

[22] F. Gul-E-Noor, M. A. Khan, S. Ghoshal, R. A. Mazid, A. M. Sarwar Uddin Chowdhury and R. A. Khan, "Grafting of 2-Ethylhexyl Acrylate with Urea on to Gelatin Film by Gamma Radiation,” Journal of Macromolecular Science, Part A, Vol. 46, No. 6, 2009, pp. 615-624. doi:10.1080/10601320902851926

[23] J. Stejskal, D. Strakova and P. Kratochvil, "Grafting of Gelatin during Polymerization of Methyl Methacrylate in Aqueous Medium,” Journal of Applied Polymer Science, Vol. 36, No. 1, 1988, pp. 215-227. doi:10.1002/app.1988.070360117 